\title{
Challenges and Countermeasures Faced by Enterprise Economic Operation Analysis in the Big Data Era
}

\author{
Yang Xin ${ }^{1}$, Yinning $\mathrm{He}^{2}$ \\ ${ }^{1}$ Institute of Financial Research, Yunnan University of Finance and Economics,Kunming-650221, P.R. China \\ ${ }^{2}$ School of Economics, Yunnan University of Finance and Economics,Kunming-650221, P.R. China
}

\begin{abstract}
The analysis of enterprise economic operation is mainly to collect, integrate, analyze and store information on the economic activities of the enterprise, so as to realize the standardization and management of the overall economic activities of the enterprise. In the era of big data, the analysis of corporate economic operations has also changed. In short, the big data environment has changed the corporate economic analysis and management mechanism, and has changed the collection of corporate operating economic data. It also has multiple impacts on data analysis and changed the overall structure of corporate economic analysis. This article will briefly discuss the challenges and countermeasures facing enterprise economic operation analysis in the era of big data, hoping to provide a reference for enterprise economic management.
\end{abstract}

\section{Introduction}

Big data combines the characteristics of global quantification, high speed and diversification, and its application value is extremely high. At the same time, the contemporary big data structure attaches great importance to the authenticity management of data information, which can judge the authenticity of data information through data identification and upgrade, making data management more valuable. With the optimization and development of the big data industry structure, the application of big data technology has become more extensive. In the current economic analysis of business operations, the application of big data technology is also extremely common. In addition, the big data environment has also changed the working mode of traditional statistical analysis. This article will briefly analyze the challenges faced by enterprise economic analysis in the era of big data, and comprehensively discuss how to do a good job in enterprise economic analysis in the era of big data.

\section{The Influence of Big Data Environment on Enterprise Economic Operation Analysis}

With the development of science and technology, the data storage, calculation and processing capabilities of computers have been greatly enhanced, and data analysis and processing service functions have been greatly improved. Internet technology can use systematic management software to effectively integrate various data in the daily management of enterprises. In this way, the scale, scope and value of data have been further extended, making the data resourceful. In business management, big data has the following characteristics.

The first feature is the huge amount of data. We can rely on computer technology and Internet technology to collect various data generated in the daily operation of the enterprise. The application of big data involves technologies such as the Internet and computers, database applications, and intelligent algorithms. We can sort, mine and analyze the data, extract relevant information from the massive and complex data information to meet actual needs. For example, Lenovo Group has been responsible for providing big data analysis for business in more than 100 countries around the world since 2018, and realizing real-time presentation of business reports. Without super data collection capabilities, such a function would not be possible. The second feature is the variety of data. In the daily operation and management of enterprises, the type of big data is not just a single "number" or "data", it should also include a series of quantifiable and non-quantifiable data resource information. Except to traditional numerical data, it also includes text, video, audio, pictures, and data sets in various forms. The third feature is the timeliness of the data. In the daily operation and management of enterprises, various data can be collected, stored, analyzed and calculated in time. Compared with traditional manual calculation and data report processing efficiency is extremely high, big data technology can effectively provide enterprises with useful economic operation information and improve enterprise management efficiency. Fourth, the value of big data is huge. Data in the traditional sense is relatively simple and contains limited value content. The value of big data is extremely high, and data is inextricably linked before. It extracts valuable information by comprehensively collecting, sorting, analyzing and merging data resources in 
enterprise production, business management, trade exchanges, etc. This can not only maximize the value of data and help companies strengthen management, but also do a good job in economic operation analysis, improve target strategies, and create greater value for the company.

\subsection{The Big Data Era's Impact on Enterprise Economic Analysis Data Collection}

The impact of big data on data collection of enterprise economic operations is reflected in three aspects. Firstly, it increases the difficulty of collecting data on the economic operation of enterprises to a certain extent. Because of the rapid growth of economic data in the context of the big data era, and the diversification of data types, the traditional single report-based data collection mode cannot meet the needs of a large number of unstructured data collection work, which will inevitably increase the difficulty of collection work. For example, M Group is a global technology group covering the four major business sectors of consumer electronics, HVAC, robotics and automation systems, and digital business. It was listed on the Fortune Global 500 list in 2016 and is currently developing strongly. The economic analysis data of the group is not only the financial statements of the previous subsidiaries and business units. Under the premise that more and more companies rely on Internet platforms to complete their product marketing in the current society, they also rely more and more on big data technology to grasp their own economic operation data in a timely manner.

Secondly, a certain degree of increase in the cost of data collection work. Compared with the data collection method in manual mode, most companies do not have a separate data department, and rely more on third-party organizations to collect the data they need. This will inevitably produce corresponding labor costs and time costs. Under the big data technology, a certain amount of labor cost has been reduced and replaced by an increase in equipment and technology costs. For example, companies need to build their own databases, servers, high-level algorithms, data collector.

Thirdly, it has improved the effectiveness of data analysis of enterprise economic operations. One of the salient features of big data technology is its effectiveness. Making full use of big data technology can quickly collect, process, and integrate and analyze massive amounts of data, extract valuable information, and quickly complete information classification and storage. For example, the business system of M Group Company is optimized and transformed, and in order to realize full-link monitoring, it is necessary to collect call logs between all business systems. More than 2 billion pieces of data are collected every day, 3 kafka clusters, and 2 logstash collection machines. In this way, the hourly and minutely data of production, warehousing, transportation, sales and management can be displayed intuitively, which is different from the previous traditional monthly and quarterly data reports.

\subsection{The Impact of Big Data on Enterprise Economic Data Processing}

The impact of the big data era on the processing of corporate economic data is manifested as a triple impact. Firstly, the data storage space has been effectively expanded. In order to meet the storage needs of a large amount of economic data and improve the efficiency of economic data processing, the staff will expand the data storage space and increase the spatial data storage capacity according to actual needs to facilitate the classification and storage of various types of massive data. For example, in 2016, M Group established a separate data back-end service department, opened up a dedicated office space as a big data server and data storage, and established a big data research and development center to provide important data support for the rapid development of the enterprise. Secondly, the efficiency of data collection and retrieval has increased to a certain extent. Because based on the big data environment, enterprise economic data presents three different types: semi-structured data, structured data and unstructured data. This brings difficulties that cannot be underestimated for the analysis and processing of enterprise economic data. In order to avoid data processing errors, the staff will design and develop different data statistical algorithms. Different algorithms use different algorithms and branch technologies to process different enterprise economic data structures in specific work, and set retrieval models by classification. The difficulty of the work can be imagined. Thirdly, it has increased the difficulty of cleaning corporate economic data. Only by cleaning up the economic data of the enterprise in real time can valuable data information be screened out, and redundant and miscellaneous can be deleted, so as to save the data needed for the economic analysis and management of the enterprise. However, in the context of the big data era, there is an explosion of massive data, and the value density of data information itself is low. We have to clean and purify all data, which will inevitably increase the difficulty of the work.

\section{Enterprise Economic Data Analysis Countermeasures in the Era of Big Data}

\subsection{Optimize Big Data Technology Functions}

In order to adapt to the development trend of the times, to deal with the challenges faced by the economic data analysis of enterprises in the era of big data, and to overcome the difficulties encountered in the current analysis work, we must fully optimize the technical functions of big data, improve the software and hardware equipment, and carry out the compatibility of the software and hardware branch systems. optimization. First of all, we should innovate economic data information collection technology methods and processing technology methods, and accelerate the update and improvement of data algorithms. We need to set up a compatible processing platform for data of different structure types, and each branch system provides appropriate analysis and processing methods and technologies at the same time. For 
example, M Group Corporation sets up odps in its own data platform, which are various calculation models. There are three main calculation models, the first is SQL, the second is mapreduce job, and the third is artificial intelligence learning job. After the analysis technology identifies the type of data structure, the branch processing technology quickly processes the data. Second, we should comprehensively improve data mining technology. Only in this way can the enterprise economic data extraction and cleaning work be done quickly, and valuable data information can be extracted in time. In addition, with the continuous development of China's Internet technology, the commercialization and coverage of $5 \mathrm{G}$ networks. The economic situation is constantly changing, and with it the scale of enterprises is also expanding. More and more companies are gradually transforming from relying on third-party big data and cloud data services to creating their own data application platforms. In the continuous development of M Group, smart chips have been added to various business and product modules. The data service platform that the group itself has spent a lot of money to build has gradually moved from meeting its own needs to the society, developing and providing data services for other companies.

\subsection{Make A Good Economic Analysis Model for Big Data Enterprises}

There are many data analysis models, and big data technology can be selected for different methods and different data types. While improving the data usage chain, its field also improves the efficiency of enterprise data analysis. Several common data analysis models of $\mathrm{M}$ group companies are as follows.

\subsubsection{Event Analysis Model}

An event refers to a behavior of a user operating a product, that is, what the user does in the product, which is translated into a descriptive language as "operation + object". Event analysis is the analysis operations such as statistics, dimensional subdivision, and screening of indicators of user behavior events. The data platform page is shown in Figure 1.

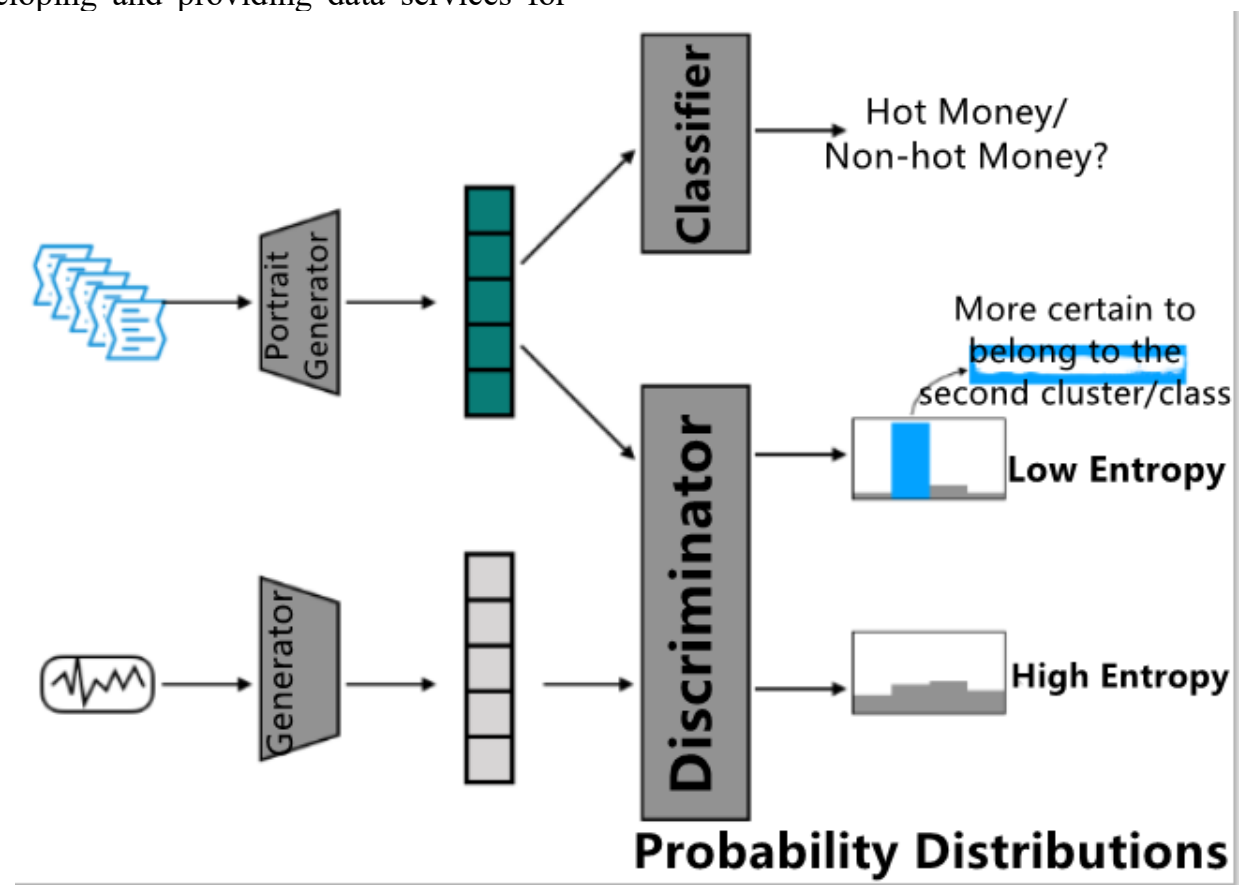

Fig1. Event Analysis Model

The company's product and operation departments can have an intuitive grasp of the overall data such as PV, UV, DAU, etc., including their values and trends every day. In the face of complex data, it is not only inefficient from the figures alone, but it is also difficult to intuitively discover the trends behind the data. This type of problem can be easily solved by using event analysis models.

\subsubsection{Model Application}

In 2016, M Group newly developed a water heater that can be connected to WIFI, and wanted to analyze and explore the user's water consumption behavior for the new water heater. Based on the user's water heater usage data collected by $\mathrm{M}$ Group through the big data platform, the corresponding mining and analysis were carried out to divide water use events and identify bathing events. Using the use of hot water as a basic event to divide and identify the provided WIFI record data can understand the distribution of users' overall use of water heaters. This helps to understand the water heater's behavior and habits among customers.

With the built-in chip of the WIFI water heater, the user data is captured and temporarily stored, and synchronized to the mobile app of the corresponding customer via WIFI, so that the data can be uploaded to the big data platform through the mobile app. The data platform analyzes customers' preferences and characteristics when using hot water. Comparing customer usage habits and customer value of different customer groups can not only deepen the understanding of customers, but also provide the most 
suitable personalized products for different customer groups. Moreover, this is conducive to the intelligent development and layout of new products, and it can also be based on formulating corresponding marketing strategies and providing APP data support.

\subsubsection{Implementation Process}

The main process of this data mining modeling is as follows: (1) Selectively extract historical data and construct expert samples. (2) Data exploratory analysis and preprocessing. (3) Establish a bathing event recognition model. (4) Divide the bathing events. (5) Application of bathing event recognition model. as shown in figure 2.

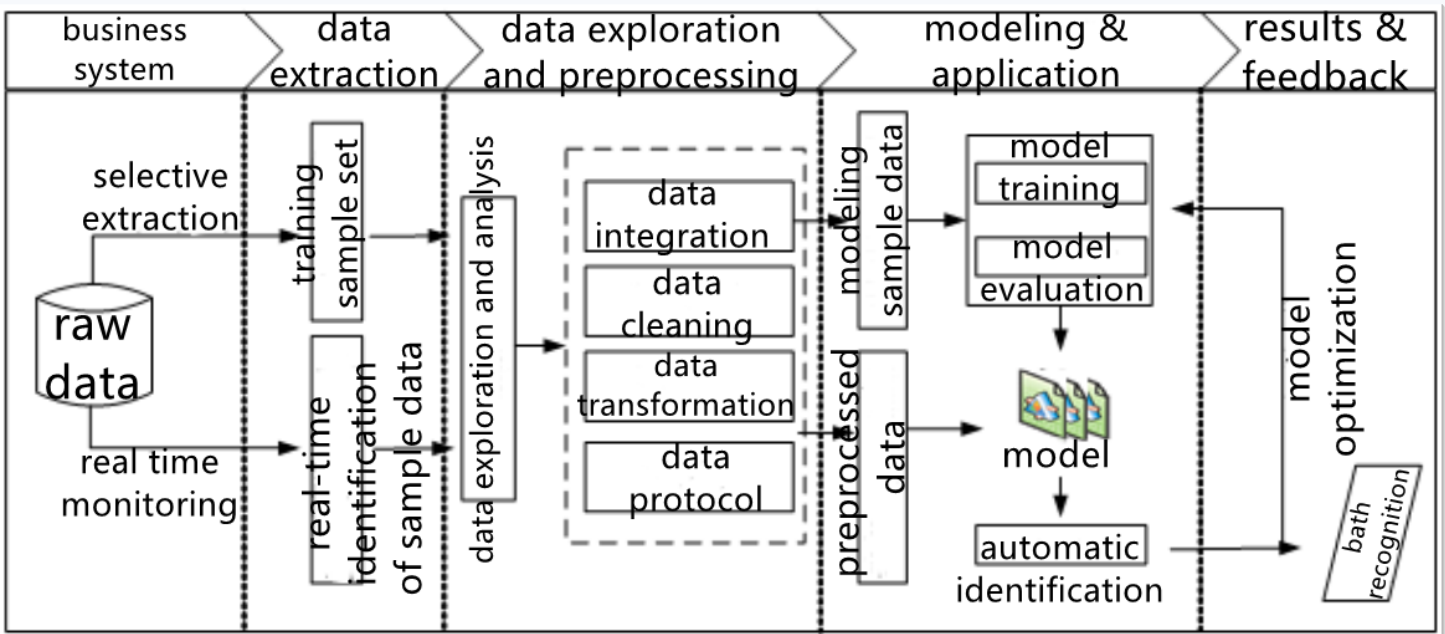

Fig2. Data Mining Modeling Process

This example analyzes the usage data of WIFI water heater users, explores the bath characteristics of water heater users, and establishes a bath recognition model. At the same time, we have established calculation models for electricity consumption and standard water consumption. Through the analysis of the water flow rate of the user's water use, the time interval of water stoppage and other indicators, the division model of the customer's water use incident was established. In the partition model, we establish a threshold optimization model for the pause time interval. For special continuous water use incidents, continuous water use identification indicators have been established for optimization. In order to improve the recognition rate of bathing events, a screening algorithm rule was developed to eliminate short-term water use events to identify "similar bathing events". The system can use the bathing events in the user's water log as basic training samples, and identify bathing events through the artificial intelligence network model. After testing, the model recognition accuracy rate reached more than $90 \%$. The calculation results of indicators such as electricity and water consumption for a single water use event are in line with the actual situation.

\subsubsection{Funnel Analysis Model}

Funnel analysis is a set of process data analysis model, which can measure the conversion effect of each node by taking each behavior node at the beginning of user behavior as an analysis model node. It is an important tool for transformation analysis. Through funnel analysis, the path of a user can be restored from first to last order, and the conversion data of each conversion node can be analyzed.

New Funnel Analysis

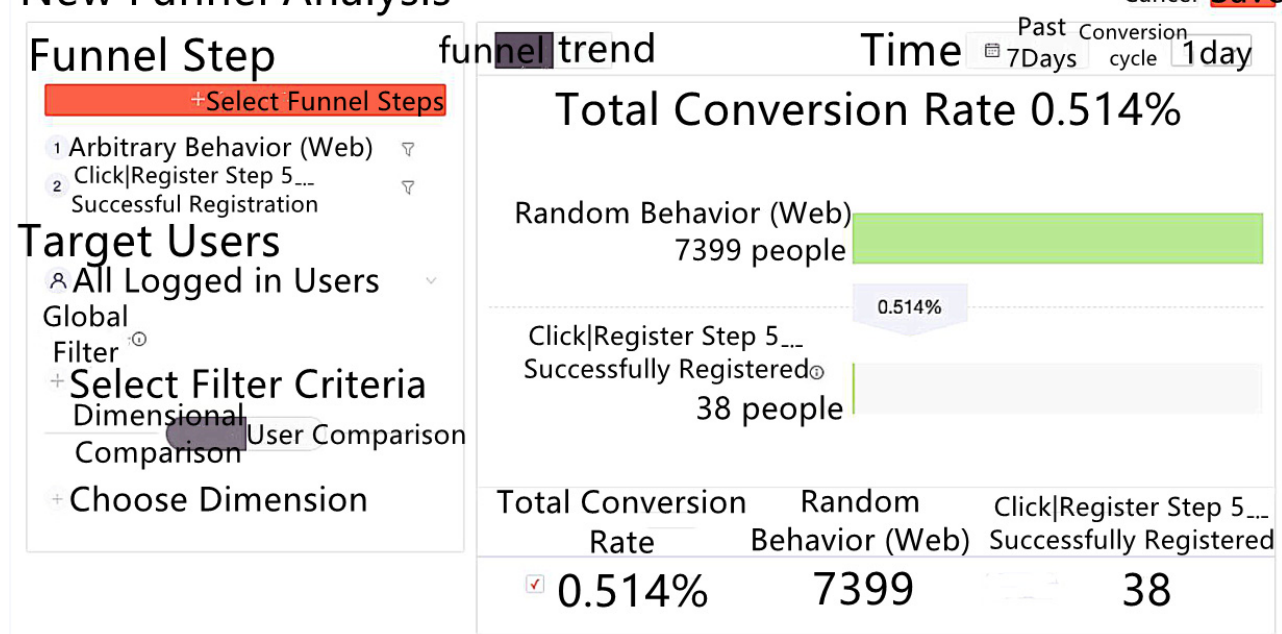

Fig3. Funnel Analysis Model 


\subsubsection{Model Application}

In 2018, during the Double 11 Shopping Festival, M Group monitored e-commerce platform users at various conversion levels, looking for optimization points at each level. And for users who did not follow the process, draw their conversion path to find the space that can improve the user experience and shorten the path. On the day of the shopping festival, users will inevitably place an order according to a pre-designed purchase process when purchasing goods, and finally complete the payment. These data are assisted by third-party e-commerce platforms, and rely on the combing of the entire business process. In the end, the system will process and analyze the output of the funnel model to find the problem of user loss at each step.

\subsubsection{Implementation Process}

Figure 4 shows the conversion rate (simulated data) of ecommerce platform users at different conversion levels.

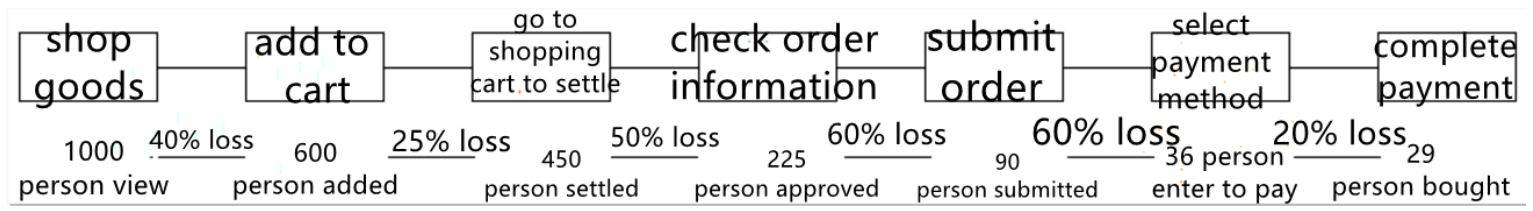

Fig4. Funnel Model Flowchart

Obviously, sometimes companies also need to do some competitive product analysis, and know the conversion of similar data in the same industry. Minimizing user churn is our goal. However, if it can be achieved that is not lower than the industry average and resources are limited, reducing the loss of users in this conversion funnel needs to be placed in a lower priority.

There are also some classic funnel conversion models that are used in the user registration process. We need to know how many users clicked the registration button (the beginning of the funnel), how many users completed the information filling (how many users gave up), how many users clicked the send verification code button (verification code arrival rate), and the number of people who successfully completed the registration. If you find that the number of registered users on a certain day fluctuates during the operation, in addition to checking the market channels and advertising, the registration function of the product itself is also an important factor that may cause this problem.

For non-functional pages of the product, such as a certain event page, company profile page, etc., users may not arrive in accordance with our established process. Users should confirm whether it is necessary to optimize the conversion process of such non-functional pages based on actual goals.

\section{Conclusion}

To sum up, in the context of the era of big data, the economic analysis staff of the enterprise view should comprehensively analyze the elements and relationships of economic data. Companies can use big data analysis methods and computer technology to re-analyze and forecast processed economic data. Enterprises need to pay attention to that unstructured data information is difficult to use the previous technical methods, which requires full use of big data technology to build a feasible data analysis mechanism. Furthermore, companies must face up to the impact of big data on economic data collection, processing, and analysis. Enterprises need to continuously improve the technical functions of big data and do a good job in the economic analysis model of big data enterprises.

\section{References}

1. Li Yan. Thoughts on strengthening business management in the era of big data $[\mathrm{J}]$, Management Aspect 2020. (34)

2. Mo Zuqin. Discussion on how financial analysis can effectively serve as a reference for business decisionmaking [J] Accounting Learning, 2020(10).

3. Chen Yong. Discussion on the challenges and measures of business management in the era of big data [J] Industrial Innovation Research, 2019 (12).

4. Fu Chao. Challenges and countermeasures of business management in the era of big data [J]. Modern State-owned Enterprise Research, 2019 (10).

5. Cao Ran, Liu Junyan. Analysis of the business management model from the perspective of big data [J] China Management Informationization 2020 (02).

6. Qu Ping. Data mining technology based on the era of big data $[\mathrm{J}]$. China's new technology and new products 2019 (04).

7. Chen Liangchen. Research on key technologies of big data mining and analysis [J]. Digital Technology and Application 2015 (011).

8. Yao Wang. The impact of big data analysis on corporate decision-making [J]. China Business Review 2019(000)002. 